\title{
Statistical Study of Solar Forcing of Total Column Ozone Variation Over Three Cities in Kenya
}

\author{
Carolyne M. M. Songa ${ }^{1,2}$, Jared H. O. Ndeda ${ }^{2}$ \& Gilbert Ouma ${ }^{3}$ \\ ${ }^{1}$ Natural Science Department (Physics), Faculty of Science, The Catholic University of Eastern Africa, Nairobi, \\ Kenya \\ ${ }^{2}$ Physics Department, Jomo Kenyatta University of Agriculture and Technology, Nairobi, Kenya \\ ${ }^{3}$ Meteorological Department, University of Nairobi, Kenya \\ Correspondence: Carolyne M. M. Songa, The Catholic University of Eastern Africa, P.O. Box 62157 - 00200 , \\ Nairobi, Kenya. Tel: 254-724-508-575. E-mail: cmutambi@cuea.edu
}

Received: March 21, 2016 Accepted: March 30, 2016 Online Published: April 30, 2016

doi:10.5539/apr.v8n3p77 URL: http://dx.doi.org/10.5539/apr.v8n3p77

\begin{abstract}
In this study, a statistical analysis between three solar activity indices (SAI) namely; sunspot number (ssn), F10.7 index (sf) and Mg II index (mg) and total column ozone (TCO) time series over three cities in Kenya namely; Nairobi $\left(1.17^{\circ} \mathrm{S} ; 36.46^{\circ} \mathrm{E}\right)$, Kisumu $\left(0.03^{\circ} \mathrm{S} ; 34.45^{\circ} \mathrm{E}\right)$ and Mombasa $\left(4.02^{\circ} \mathrm{S} ; 39.43^{\circ} \mathrm{E}\right)$ for the period $1985-$ 2011 are considered. Pearson and cross correlations, linear and multiple regression analyses are performed. All the statistical analyses are based on $95 \%$ confidence level. SAI show decreasing trend at significant levels with highest decrease in international sunspot number and least in Mg II index. TCO are highly correlated with each other at $(0.936<\mathrm{r}<0.955, \mathrm{p}<0.001)$. SAI are also highly correlated with each other at $(0.941<\mathrm{r}<0.976, \mathrm{p}<$ 0.001 ) and are significantly positively correlated with TCO over the study period except Mg II index at Kisumu. TCO and SAI have correlations at both long and short lags. At all the cities, F10.7 index has an immediate impact and Mg II index has a delayed impact on TCO. A linear relationship exists between the two variables in all the cities. An increase in TCO of about $2-3 \%$ (Nairobi), $1-2 \%$ (Kisumu) and $3-4 \%$ (Mombasa) is attributed to solar activity indices. The multiple correlation coefficients and significant levels obtained show that 3 $-5 \%$ of the TCO at Nairobi, Kisumu and Mombasa can be predicted by the SAI.
\end{abstract}

Keywords: correlation, F10.7 index, Mg II index, regression, solar activity indices, sunspot number, time series, total column ozone

\section{Introduction}

The Sun, undergoes changes characterized by variations in its output ranging from minutes to months and decades due to the different degrees of activity (Lockwood, 2004) and these variations reflect the inhomogeneous emission of radiation due to the presence and absence of active regions on the solar disk. The variations occur because of the changing impacts of the solar features whose opposing influences depend on the wavelengths. The three manifestations of solar variability are variations of solar structures, electromagnetic radiations and energetic solar particles. Variations of the solar electromagnetic radiation are the cause of the radiated solar output variability both total and in various wavelengths (Spruit, 2000).

The magnetic fields generated by a self-exciting magneto hydrodynamics (MHD) dynamo in the interior of the sun controls the solar activity (Charbonneau, 2005). The distinctive features perpetrated by these magnetic fields are displayed on different solar spheres, namely, the photosphere, chromosphere, and corona. Even though different solar layers are coupled, the variability is strongly wavelength dependent.

Solar cycle variations affect stratospheric ozone through changes in the UV fluxes that affects the dissociation of chemical species. In particular, the solar Ultraviolet (UV) radiation plays a major role in the temperature, dynamics and photochemistry of the stratosphere (Newman, 2004; Gray, Rumbold, \& Shine, 2009; Gray, 2010). UV radiation between 120 and $300 \mathrm{~nm}$ is absorbed in the earth's outer atmosphere by ozone and molecular oxygen. Increased levels of UV radiation heat the stratosphere and mesosphere due to absorption by ozone and oxygen. Therefore variations in the different spectral regions can have a major impact on the Earth's atmosphere 
(Geller., 1988; Marsh et al., 2007) since the origins and fate of life on Earth are intimately connected to the way different layers of the Earth's atmosphere respond to these variations (Tsiropouli, 2003).

Many different indicators have been employed to measure solar activity (Lean, 2000b). The sunspots, visible on the solar disk at any given time, has been the most commonly used parameter and the basic indicator of solar activity hence, a proxy for the general state of solar activity. Since no single proxy can produce the solar variability over the whole UV spectrum various other indicators and proxies for various UV spectral bands that encompass emissions coming from the solar corona down to the photosphere do exist (Dudok, Kretzschmar, Lilensten, \& Woods, 2009) and have been tested (Dudok \& Watermann, 2007).

Relative sunspot numbers are the collection of sunspot numbers that provide spot counts averaged over different time intervals. It is given by the sum of the number of individual sunspots and ten times the number of groups. On average, most sunspot groups have about ten spots; hence this formula for counting sunspots gives reliable numbers even when the observing conditions are less than ideal and small spots difficult to observe (Jana, Bhattacharyya, \& Midya, 2013)

The 10.7-cm solar radio flux, F10.7 was first observed by Covington (1948). F10.7 index is the solar output originating in the high solar atmospheric layers of the chromosphere and in lower corona. The solar flux, measured in solar flux units (Solar Flux Unit [SFU], Tobiska, Bouwer \& Bowman, 2008), is the amount of radio noise or flux emitted at a frequency of $2800 \mathrm{MHz}(10.7 \mathrm{~cm})$. Representing a measure of diffuse, non-radiative heating of the coronal plasma trapped by magnetic fields over the active regions, the F10.7 index is an excellent indicator of overall solar activity levels in the UV region. Each value of F10.7 index is a measurement of the total emission at a wavelength of $10.7 \mathrm{~cm}$ from all sources present on the solar disk, made over a $1 \mathrm{~h}$ period centered on the epoch given for the value (Tapping, 2013)

The Mg II core-to-wing index has been shown to be a good measure of solar chromospheric activity for solar features and wavelengths that have strong chromospheric components (Viereck et al., 2001). It is a ratio of the Mg II chromospheric emission at $280 \mathrm{~nm}$ to the photospheric radiation in the line wings (de Toma, White, Knapp, Rottman, \& Woods, 1997). Introduced by Heath and Schlesinger (1986), the Mg II core-to-wing ratio is one of the most widely used indices of solar activity in the UV region. The Mg II core to wing ratio is calculated by taking the ratio between the highly variable chromospheric Mg II h and k lines at 279.56 and $280.27 \mathrm{~nm}$ respectively and weakly varying wings or nearby continuum which are photospheric in origin. The result is mostly a measure of chromospheric solar active region emission that is theoretically independent of instrument sensitivity change through the time (Bruevich, Bruevich, \& Yakunina, 2013)

Several studies, for example, (Angell, 1989; Labitzke \& Van Loon, 1997; Isikwue, Agada, \& Okeke, 2010) have shown increase in solar ultraviolet radiation with increase in sunspot number and also a more or less in phase variation of TCO with sunspot number. The relationship between ozone concentration and sunshine in Nigeria carried out by Obiekezie (2009) for a period 1997 - 2005 using linear regression analysis gave a significant negative correlation between the two parameters. The linear regression analysis carried out by Rabiu and Omotosho (2003) to determine the relationship between solar activity and TCO variation in Lagos again in Nigeria, showed a significant negative correlation between mean total column ozone and solar activity both at monthly level and annual level, showing that the total column ozone decreases with increasing solar activity.

Isikwue, Agada and Okeke (2010) performed a multiple regression analysis in their study on the contribution of solar activity indices on the stratospheric ozone variations in some cities in Nigeria from 1998 - 2005 and obtained a positive significant correlation between sunspot numbers and ozone concentration and negative nonsignificant correlation between $\mathrm{Mg}$ II core to wing ratio and ozone concentration.

A statistical relationship (Pearson product moment correlation coefficient) between surface ozone and solar activity in a tropical rural site on the east coast of south India (1996 - 2004) investigated by Selvaraj, Gopinath, \& Jayalakshmi (2010b), found a high positive rank correlation coefficient of 0.76 and 0.62 obtained for the years 2000 and 2002 respectively indicating the influence of higher solar activity over the surface ozone levels.

A study on the relationship between ozone and solar activity has not been carried out in cities and towns in Kenya. Hence to give an idea of solar activity effect in this low latitude region, the contributions or inputs of the solar activity indices namely; the international sunspot number (index of the sun's photosphere), the $10.7 \mathrm{~cm}$ solar radio flux from the sun's corona and the Mg II core-to-wing ratio (index of the sun's chromospheres), are considered for the sun's influence on the stratospheric ozone in Kenyan cities namely Nairobi, Kisumu and Mombasa using statistical analysis. 


\section{Materials and Methods}

\subsection{Data}

The dataset used in this study consists of the daily values of TCO (at Nairobi, Kisumu and Mombasa) and SAI namely, international sunspot number (ssn), F10.7 cm solar radio flux (sf) and Mg II core to wing ratio (mg).

\subsubsection{Total Column Ozone Dataset}

A near-global TCO database at $1.25^{\circ}$ longitude by $1^{\circ}$ latitude, combining measurements from multiple satellitebased instruments (Bodeker, Hassler, Young, \& Portmann, 2013) has been used to interpolate data to the three locations of interest in Kenya. The Bodeker scientific database extends from $1^{\text {st }}$ November 1978 to 2013 and can be obtained from data site: http://www.bodekerscientific.com/data/total-column-ozone. Comparisons against TCO measurements from the ground-based Dobson and Brewer spectrophotometer network are used to remove offsets and drifts between a subset of the satellite-based instruments. The corrected subset is then used as a basis for homogenizing the remaining data sets.

\subsubsection{Solar Activity Indices Dataset}

These datasets are selected for the period January1985 to December 2011 from existing data sources; The independent variable of solar indices are obtained from data sites as follows; The daily international sunspot number which is the photospheric index are taken from data site http://www.ngdc.noaa.gov/stp/ space-weather/solar-data/solar-indices/sunspot-numbers/international/listings/listing_international-sunspot-numb ers daily.txt the $\mathrm{F} 10.7 \mathrm{~cm}$ solar radio flux which is the coronal index are obtained from data site: ftp://ftp.ngdc.noaa.gov/STP/space-weather/solar-data/solar-features/solar-radio/noontime-flux/penticton/penticto n_observed/ flux-observed_daily.txt and Mg II core-to-wing ratio, which is the chromospheric index are taken from data site: ftp://ftp.ngdc.noaa.gov/STP/SOLAR_DATA/SOLAR_UV/NOAAMgII.dat.

\subsection{Study Area Descriptions}

Kenya is located in the Eastern part of Africa $\left(5^{\circ} \mathrm{N}, 4.40^{\circ} \mathrm{S}\right.$ and $\left.33.53^{\circ} \mathrm{E}, 41.56^{\circ} \mathrm{E}\right)$ and has a total area of 582,646 $\mathrm{km}^{2}$. Table 1 shows the codes, positions and elevations of the three Kenyan cities.

Table 1. Names, codes, position and elevation of the three Kenyan cities

\begin{tabular}{lcccc}
\hline Station & Code & Latitude $\left({ }^{\circ} \mathrm{S}\right)$ & Longitude $\left({ }^{\circ} \mathrm{E}\right)$ & Altitude (m, asl) \\
\hline Nairobi & nrb & 1.17 & 36.46 & 1661 \\
Kisumu & $\mathrm{ksm}$ & 0.03 & 34.45 & 1131 \\
Mombasa & $\mathrm{msa}$ & 4.02 & 39.43 & 17 \\
\hline
\end{tabular}

asl $=$ above sea level.

Nairobi is centrally located in the country and covers an area of $684 \mathrm{~km}^{2}$. It is the country's capital and largest city and holds a population of 3.1 million people (Kenya National Bureau of Statistics [KNBS], 2010). Mombasa is Kenya's second largest city, located on the South Eastern coast of the country, along the Indian Ocean. The city has an area of $295 \mathrm{~km}^{2}$ and a population of 939370 people (KNBS, 2010). Kisumu is the third largest city in Kenya. It is located in Western Kenya and covers an area of approximately $417 \mathrm{~km}^{2}$, with a population of 968 909 people (KNBS, 2010).

\subsection{Methodology}

Data reduction is done to get the mean monthly values of both TCO at each location and the SAI variables. For data to represent the monthly and yearly scales, means and the respective standard deviation for the concerned time periods are calculated. The monthly means of the daily values of the SAI and TCO is evaluated by taking the averages of all the days in every month for each year of the data. The statistical relationship between SAI and TCO over the period 1985 to 2011 is examined in Nairobi, Kisumu and Mombasa. The R software version 3.2.3 is employed to obtain the results.

Time series plots of mean monthly of both TCO at Nairobi, Kisumu and Mombasa and the SAI variables are shown in Figure 1. A descriptive statistical summary including TCO in the various locations and SAI variables (ssn, sf and $\mathrm{mg}$ ) along with their attributes are produced in Table 2 and Table 3 respectively 
Analysis is carried out in four aspects namely; Pearson correlation analysis, cross correlation analysis, linear and multiple regression analyses. All statistical analyses are based on $95 \%$ confidence level where $\mathrm{p}$ value or significant value is less than 0.05 for the result to be significant.

\subsubsection{Pearson Correlation Analysis}

Pearson correlation analysis is conducted to examine the relationship between monthly mean SAI variables ssn, sf and mg and mean monthly TCO at each city over the study period. Correlations among SAI variables and among TCO are also performed.

\subsubsection{Cross Correlation Analysis}

The cross correlation function between the time series of both mean monthly TCO at each city and SAI variables is analyzed to determine the time lag(s) of SAI preceding TCO at which the series showed strongest correlations.

\subsubsection{Linear Regression Analysis}

The time lags with maximum correlation coefficient values are used in the linear regression of SAI and TCO to obtain linear relationships between them. The linear dependence significance is justified by the $t-$ test and further confirmed by the analysis of variance (ANOVA) results.

\subsubsection{Solar Forcing Effect}

SAI variables are employed to model TCO at Nairobi, Kisumu and Mombasa by using a linear multivariate model and applying the least square fittings. Table 10 presents the values of multiple correlation coefficients obtained and their level of significance. The models are subjected to test using a 20 month data SAI values from January 2012 - August 2013 The correlation between the predicted and observed values of the models at $\alpha=$ 0.05 are given in Table 11 .

\section{Results and Discussions}

\subsection{Descriptive Statistics Analysis}

The various statistical attributes of both mean monthly TCO and SAI variables during the study periods are shown in Table 2 for TCO and Table 3 for SAI variables.

Table 2. Descriptive Statistics of mean monthly TCO at Nairobi (nrb), Kisumu (ksm) and Mombasa (msa) between January 1985 and December 2011

\begin{tabular}{llllll}
\hline Variable & mean & SD & Range & Maximum & Minimum \\
\hline nrb & 254.75 & 9.76 & 47.39 & 277.93 & 230.55 \\
ksm & 253.92 & 9.54 & 45.38 & 275.85 & 230.47 \\
msa & 257.07 & 8.84 & 41.77 & 277.86 & 236.09 \\
\hline
\end{tabular}

Table 3. Descriptive Statistics of mean monthly SAI variables between January 1985 and December 2011

\begin{tabular}{llllll}
\hline Variable & mean & SD & Range & Maximum & Minimum \\
\hline ssn & 57.77 & 50.81 & 200.00 & 200.00 & 0.00 \\
sf & 117.82 & 49.02 & 182.35 & 247.20 & 64.86 \\
$\mathrm{mg}$ & 0.271 & 0.006 & 0.025 & 0.2880 & 0.263 \\
\hline
\end{tabular}

\subsection{Trend Analysis}

The monthly mean TCO time series from 1985 - 2011 are shown in Figures 1 (a) - (c) and the mean monthly SAI time series for the same period are shown in Figures 1(d) - (f). Songa, Ndeda and Ouma (2015) discussed in details the TCO trends and variability in Nairobi, Kisumu and Mombasa.

A decrease in ssn, sf and $\mathrm{mg}$ with time is observed. The decrease could be due to declining solar activity since all the indices of solar activity are in most cases closely related as the main source of all their variations is variable magnetic field. The trends are different but at significant levels for each SAI variable as shown by the linear fits in Table 4 . The linear trends suggest that, over the 27 year period of study, a decrease of $94 \%$ of the mean for ssn, 
$34 \%$ of the mean for sf and $\approx 2 \%$ of the mean for mg. Hence there is a decreasing trend of SAI variables showing that during the study period, SAI is decreasing with highest decrease in ssn and least in mg.
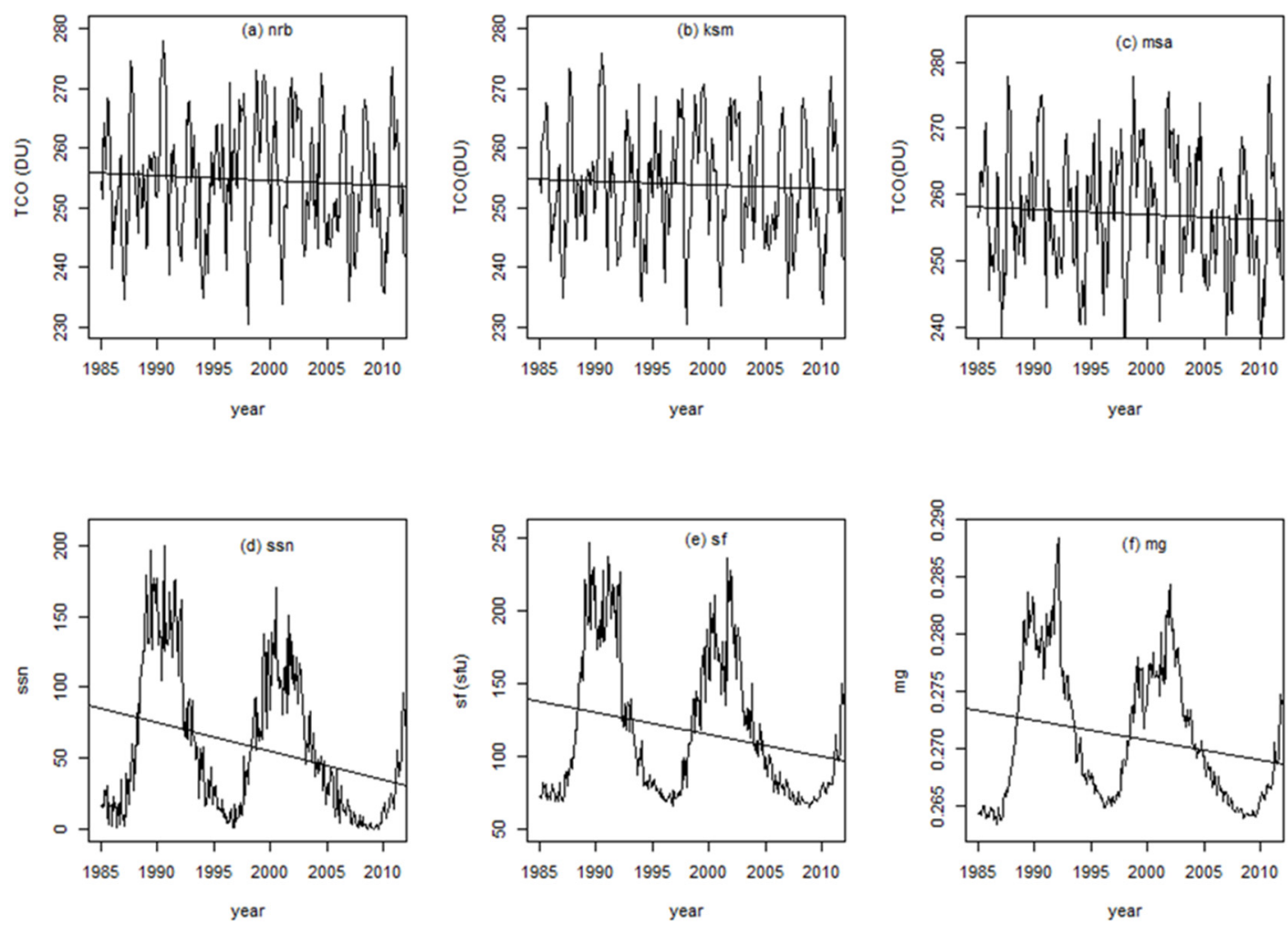

Figure 1. Mean monthly time series for TCO at (a) Nairobi (nrb), (b) Kisumu (ksm), (c) Mombasa and SAI variables (d) international sunspot number (ssn), F10.7 cm solar radio flux (sf) and $\mathrm{Mg}$ II core to wing ratio (mg) from 1985 - 2011 with their linear fits

Table 4. Trends derived from linear fits to the mean monthly TCO and SAI time series from $1985-2011$

\begin{tabular}{llll}
\hline Variables & Trend/yr. & $\mathrm{r}^{2}$ & $\mathrm{p}$ values \\
\hline TCO $(\mathrm{nrb})$ & $-0.0799 \pm 0.06953 \mathrm{DU}$ & 0.004084 & 0.2514 \\
TCO $(\mathrm{ksm})$ & $-0.0651 \pm 0.06800 \mathrm{DU}$ & 0.002852 & 0.3379 \\
TCO $(\mathrm{msa})$ & $-0.08016 \pm 0.06297 \mathrm{DU}$ & 0.005014 & 0.2037 \\
$\mathrm{ssn}$ & $-2.0154 \pm 0.3449$ & 0.09587 & $<0.001$ \\
$\mathrm{sf}$ & $-1.4968 \pm 0.3399 \mathrm{sfu}$ & 0.05681 & $<0.001$ \\
$\mathrm{mg}$ & $-0.0001726 \pm 0.0004 .183$ & 0.05084 & $<0.001$ \\
\hline
\end{tabular}

\subsection{Pearson Correlation Analysis}

Pearson correlation analyses are performed relating mean monthly TCO at the three cities, monthly SAI variables and mean monthly TCO at the three cities to monthly SAI variables. Figure 2 shows the distribution of each variable on the diagonal. On the bottom of the diagonal, the bivariate scatter plots with a fitted line are displayed while on the top of the diagonal, the value of the correlation and the significance level as stars are displayed. 


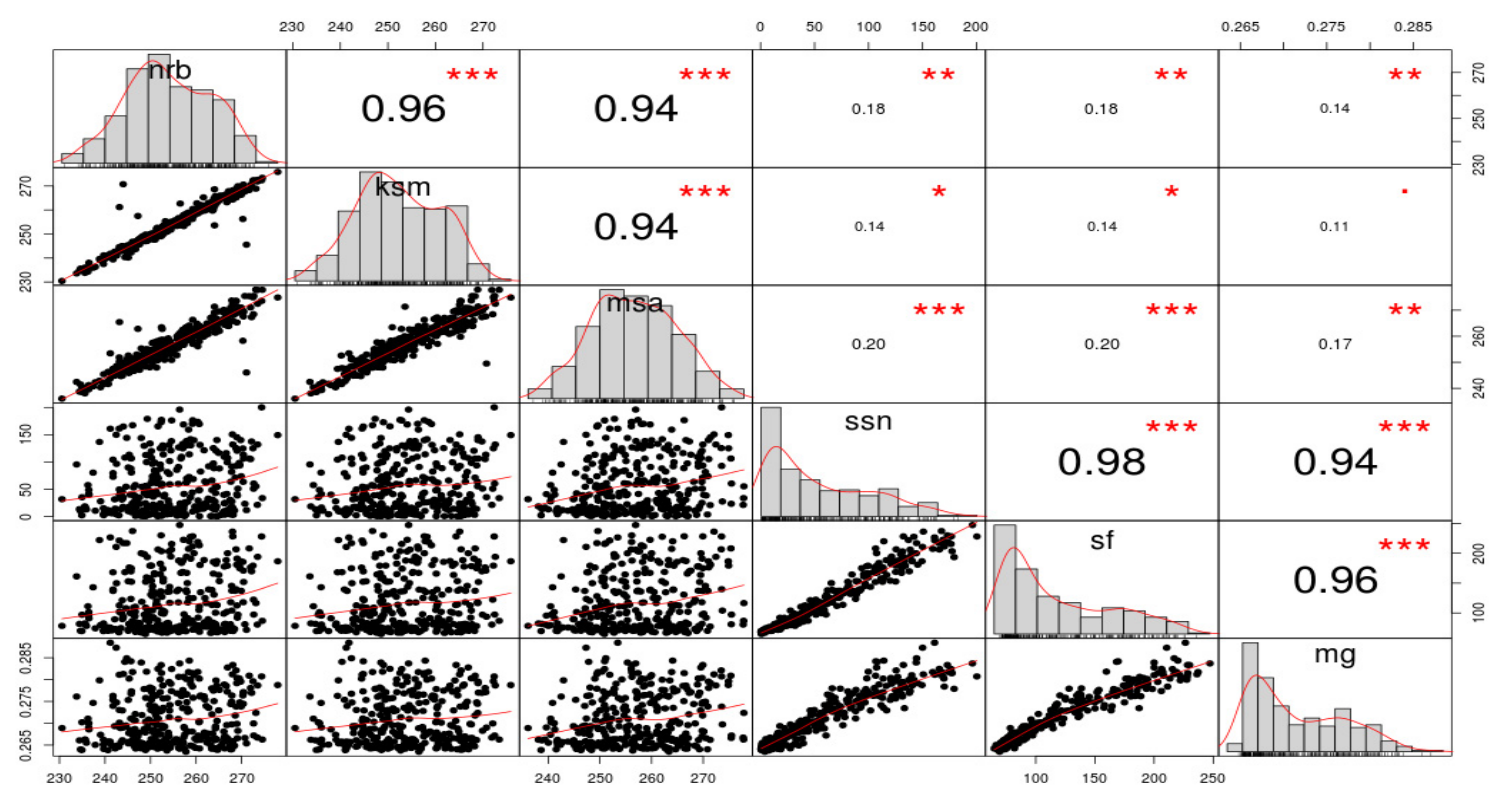

Figure 2. Correlation matrix of TCO (nrb, ksm and msa) and the SAI variables (ssn, sf and mg)

P-values $(0,0.001,0.01,0.05,0.1,1)<=>$ symbols (“****, “**”, “*”, “.”, " “) , 2 - tailed.

Pearson's correlation analysis demonstrate that TCO at Nairobi, Kisumu and Mombasa are highly correlated with each other at $\mathrm{p}<0.001$ (2- tailed) significant level. The highest correlation of 0.955 is found to exist between TCO at Nairobi and Kisumu (Table 5). A lowest correlation coefficient of 0.936 is observed between TCO at Nairobi and Mombasa among the cities.

Table 5. Association among mean monthly TCO at the three cities for the period $1985-2011$ and significant level

\begin{tabular}{llllll}
\hline & TCO $(\mathrm{nrb})$ & $\mathrm{p}$ & $\mathrm{TCO}(\mathrm{ksm})$ & $\mathrm{p}$ & TCO $(\mathrm{msa})$ \\
\hline TCO $(\mathrm{nrb})$ & 1 & & & & \\
TCO $(\mathrm{ksm})$ & 0.955 & $<0.001$ & 1 & & \\
TCO $(\mathrm{msa})$ & 0.936 & $<0.001$ & 0.938 & $<0.001$ & 1 \\
\hline
\end{tabular}

The association among the SAI variables is shown in Table 6. SAI variables are also highly correlated with each other at $\mathrm{p}<0.001$ (2- tailed) significant level with highest correlation coefficient for the association between ssn and $\operatorname{sf}(\mathrm{r}=0.976, \mathrm{p}<0.001)$. The association between ssn and $\mathrm{mg}(\mathrm{r}=0.941, \mathrm{p}<0.001)$ is the least. $\mathrm{sf}$ and $\mathrm{mg}$ has a correlation of $(r=0.960, p<0.001)$.

Table 6. Association among mean monthly SAI variables (ssn, sf and mg) for the period $1985-2011$ and the level of significance

\begin{tabular}{llllll}
\hline & ssn & p & sf & p & mg \\
\hline ssn & 1 & & & & \\
sf & 0.976 & $<0.001$ & 1 & & \\
mg & 0.941 & $<0.001$ & 0.960 & $<0.001$ & 1 \\
\hline
\end{tabular}

These results agree well with Pearson's correlation coefficient between F10.7 cm solar radio flux and international sunspot number values range between 0.94 and 0.98 (Tharshini \& Shanthi, 2015). Hathaway et al., (2003) reported that a correlation between F10.7 cm solar radio flux and relative sunspot number exceeds 0.98 . 
The high correlation coefficient between F10.7 $\mathrm{cm}$ solar radio flux and international sunspot number and also between F10.7 cm solar radio flux and Mg II core-to-wing ratio could be due to the fact that F10.7 cm solar radio flux comes from high part of chromosphere and lower part of corona of the sun where it tracks other important emissions that form in the same region of the solar atmosphere (Tapping, 2013). High degree of correlation of F10.7 cm solar radio flux and Mg II core-to-wing ratio suggests dependency upon common plasma parameters and that their sources are spatially close. High correlation between international sunspot number and $\mathrm{Mg}$ II core-to-wing ratio could be due to the $280 \mathrm{~nm} \mathrm{Mg}$ II solar spectral band containing photospheric continuum and chromospheric line emission hence chromospheric in origin while weakly varying wings or nearby continuum are photospheric in origin.

The monthly averaged ssn, monthly averaged sf and monthly averaged mg (Table 7), correlations though low, are significantly positively correlated with monthly mean TCO over the study period except for mean monthly TCO and averaged $\mathrm{mg}$ at Kisumu where $\mathrm{p}>0.05$ hence insignificant. Among the SAI variables, sf is most closely correlated to monthly TCO at $\mathrm{nrb}(\mathrm{r}=0.1753, \mathrm{p}<0.05)$ and Mombasa $(\mathrm{r}=0.2039, \mathrm{p}<0.001)$ while ssn is most correlated to monthly TCO at Kisumu $(r=0.1372, p<0.05$. Mg was least correlated to monthly TCO at nrb $(\mathrm{r}=0.1440, \mathrm{p}<0.05)$, at $\mathrm{ksm}(\mathrm{r}=0.1089, \mathrm{p}>0.05)$ and at msa $(\mathrm{r}=0.1740, \mathrm{p}<0.05)$. Correlation of SAI and ozone is due to the fact that most of the solar EUV radiation is absorbed in the upper terrestrial atmosphere some of which include international sunspot number, F10.7 cm solar radio flux and Mg II core-to-wing ratio. Correlation in the equatorial region is low where ozone is produced and in the sub Polar Regions where the larger amounts of ozone is found. The effect could be, according to Labitzke and Van Loon (1997) due to solar induced changes on the pole ward transport of ozone rather than of due to radiative interaction between sun and ozone (Tsiropoula, 2003)

Table 7. Association between mean monthly TCO and mean monthly SAI variables for the period $1985-2011$ and their level of significance

\begin{tabular}{lllllll}
\hline & TCO $(\mathrm{nrb})$ & $\mathrm{p}$ & $\mathrm{TCO}(\mathrm{ksm})$ & $\mathrm{p}$ & $\mathrm{TCO}(\mathrm{msa})$ & $\mathrm{p}$ \\
\hline ssn & 0.1750 & 0.001 & 0.1372 & 0.05 & 0.1974 & $<0.001$ \\
sf & 0.1753 & 0.001 & 0.1368 & 0.05 & 0.2039 & $<0.001$ \\
$\mathrm{mg}$ & 0.1440 & 0.001 & 0.1089 & 0.10 & 0.1740 & 0.001 \\
\hline
\end{tabular}

Hence Pearson analysis demonstrates that TCO at all the cities are highly correlated among each other at $\mathrm{p}<$ 0.001 (2- tailed) significant level. All correlations are positive and highest correlation is found to exist between TCO at Nairobi and TCO at Kisumu. SAI variables are also positive and highly correlated among each other with the highest correlation coefficient $(0.976)$ between ssn and sf. SAI variables are found to bear a significant correlation with mean monthly TCO at Nairobi and Mombasa at $\mathrm{p}<0.05$ except at Kisumu.

\subsection{Cross Correlation}

Cross correlation function between the time series of TCO at each city and SAI variables is performed and the time lags of SAI preceding TCO at which the series showed strongest (maximum) and minimum correlation is then determined at different cities as shown in Table 8.

ssn exhibited the same maximum lag month 0 at Nairobi $(r=0.175)$ and Mombasa $(r=0.197)$ but a different maximum correlation coefficient $(\mathrm{r}=0.140)$ at lag month 11 at Kisumu. A minimum correlation coefficient of 0.112 and 0.139 are observed between ssn and TCO at Nairobi and Mombasa respectively at lag month 6. A minimum correlation of 0.0870 at lag month 5 is observed at Kisumu. sf exhibited the same maximum at lag month 0 at all the cities while a local minimum varied between lags 4 to $6 . \mathrm{mg}$ increases gradually from lag 0 to a maximum value at lag month 9 and then drops to its minimum value at lag month 12 (Figure $3-4$ ). mg showed a constant local maximum and minimum cross correlation at lag months 9 and 12 respectively at all the cities.

The lag months for ssn and sf (Table 8) are shorter $(\leq-6)$ than lag months for mg (- 9 and - 12), except in Kisumu where a large lag month of - 11 is observed to give a maximum correlation of 0.140 between TCO and ssn. Hence sf has an immediate impact on TCO at all the cities while ssn has immediate impact on TCO at Nairobi and Mombasa. But a delayed impact at Kisumu. mg has a delayed impact on TCO at all the cities. This could also have an effect on the ozone variability. 
Table 8. Cross correlation coefficients and lags (in months) between SAI and TCO

\begin{tabular}{llcccccc}
\hline TCO & & ssn & lag/months & sf & lag/months & mg & lag/months \\
\hline nrb & Max & 0.175 & 0 & 0.175 & 0 & 0.167 & -9 \\
& Min & 0.112 & -6 & 0.123 & -6 & 0.123 & -12 \\
\multirow{2}{*}{ ksm } & Max & 0.140 & -11 & 0.137 & 0 & 0.150 & -9 \\
& Min & 0.0870 & -5 & 0.0980 & $-4,-5$ & 0.108 & -12 \\
\multirow{2}{*}{ msa } & Max & 0.197 & 0 & 0.204 & 0 & 0.190 & -9 \\
& Min & 0.139 & -6 & 0.140 & -5 & 0.142 & -12 \\
\hline
\end{tabular}

Figures 3, 4 and 5 shows the local maximum and minimum of the lagged cross correlation monthly mean TCO at respectively Nairobi, Kisumu and Mombasa and the SAI over the study period. ssn and sf patterns are similar. Both start at a higher coefficient value at zero month then drop gradually up to lag months; - 6 at Nairobi, - 4 and - 5 at Kisumu and 5 at Mombasa. The correlation starts to increase up to lag month - 10 and again decreases. The difference in the patterns could be due to decreased accuracy during solar minimum which is exhibited by ssn and sf, not $\mathrm{mg}$. Also, $\mathrm{mg}$ is a ratio and hence less sensitive to artifacts and instrumental degradation than a non-ratio measurement (Suess, Snow, Viereck \& Machol, 2016)

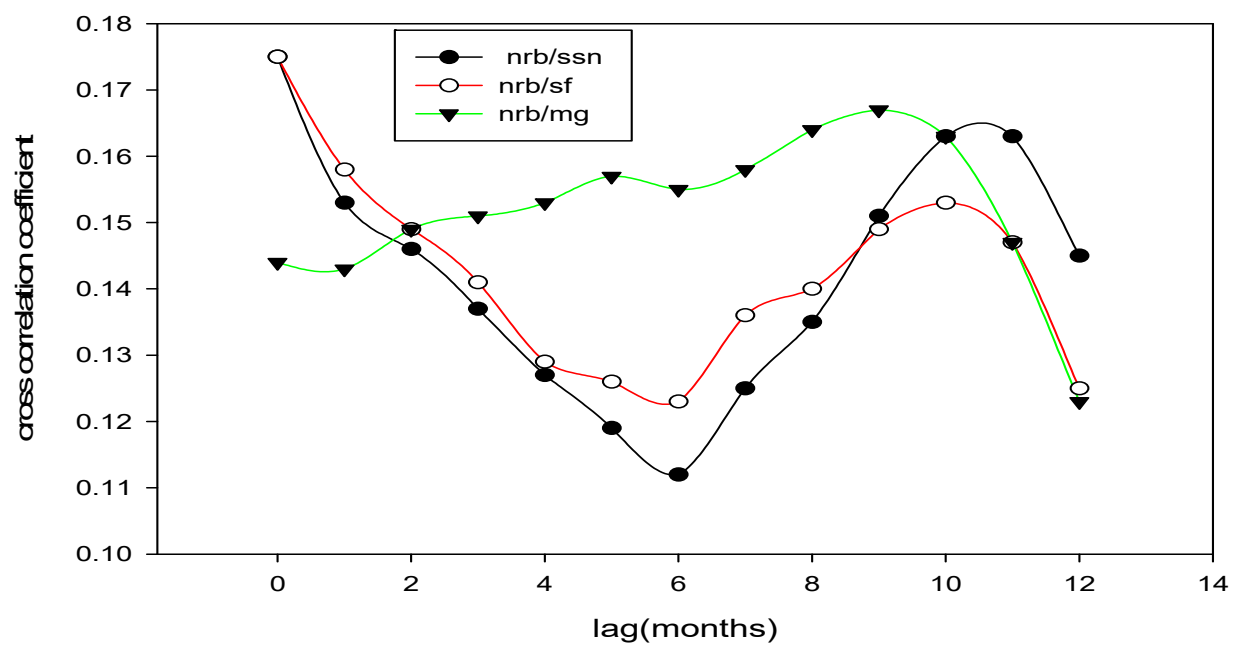

Figure 3. Plots of lagged cross correlation of monthly mean TCO at Nairobi and averaged SAI variables over the study period $1985-2011$

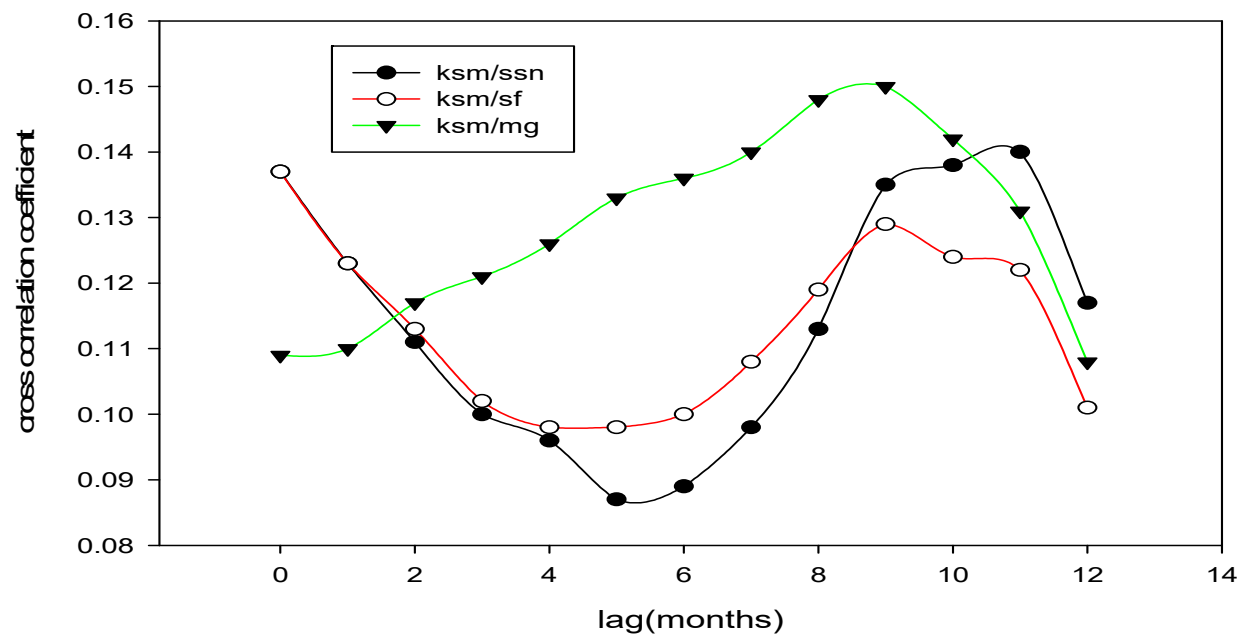

Figure 4. Plots of lagged cross correlation of monthly mean TCO at Kisumu and averaged SAI variables over the study period $1985-2011$ 


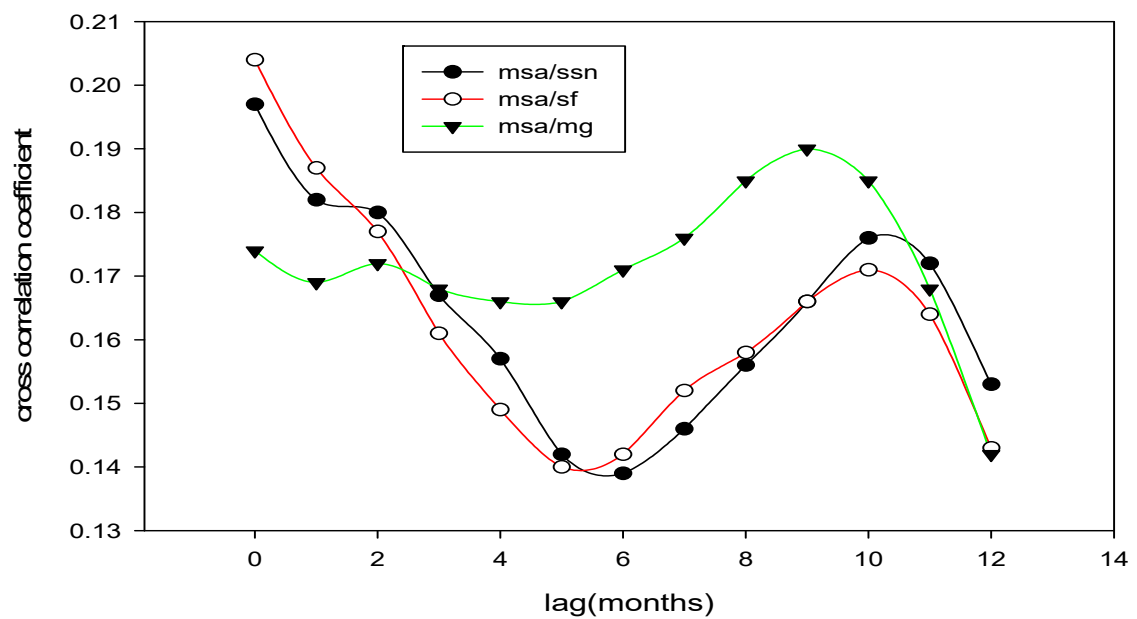

Figure 5. Plots of lagged cross correlation of monthly mean TCO at Mombasa and averaged SAI variables over the study period $1985-2011$

Hence the mean monthly TCO and mean monthly SAI variables have correlations at both long and short lags. Positive maximum correlation is again observed between TCO at all the cities and SAI variables. A local maximum cross correlation between mean monthly TCO and mean monthly SAI variables is observed when ssn and sf variables are at month zero lag with TCO at all the cities as shown in Figure 3 for Nairobi, Figure 4 for Kisumu and Figure 5 for Mombasa. A lag month of -9 is observed between mg and TCO at all the cities to give a maximum correlation.

\subsection{Linear Regression Analysis}

Tables 9(a) - (c) shows the results attained after subjecting both the mean monthly TCO at the three cities and SAI variables to linear regression. Linear regression analysis gave the correlation coefficient between TCO and SAI variables as $0.1750,0.1753$ and 0.1671 for ssn, sf and $\mathrm{mg}$ respectively at Nairobi, as $0.1372,0.1368$ and 0.1499 for ssn, sf and $\mathrm{mg}$ at Kisumu respectively and as $0.1974,0.2043$ and 0.1894 for ssn, sf and $\mathrm{mg}$ respectively at respectively at Mombasa.

Table 9(a). Results of linear regression and t-test for mean monthly TCO and mean monthly SAI at Nairobi

\begin{tabular}{lllllll}
\hline TCO & intercept & slope & $\mathrm{r}$ & $\mathrm{r}^{2}$ & $\mathrm{t}$ value & $\operatorname{Pr}(>|\mathrm{t}|)$ \\
\hline ssn & 252.81051 & 0.03362 & 0.1750 & 0.03063 & 3.190 & $0.00156^{* *}$ \\
$\mathrm{sf}$ & 250.64016 & 0.03490 & 0.1753 & 0.03073 & 3.195 & $0.00153^{* *}$ \\
$\mathrm{mg}$ & 181.760 & 268.620 & 0.1671 & 0.02793 & 2.999 & $0.00293^{* *}$ \\
\hline
\end{tabular}

Table 9(b). Results of linear regression and t-test for mean monthly TCO and mean monthly SAI at Kisumu

\begin{tabular}{lllllll}
\hline TCO & intercept & slope & $\mathrm{r}$ & $\mathrm{r}^{2}$ & $\mathrm{t}$ value & $\operatorname{Pr}(>|\mathrm{t}|)$ \\
\hline ssn & 252.42988 & 0.02576 & 0.1372 & 0.01882 & 2.486 & $0.0134^{*}$ \\
$\mathrm{sf}$ & 250.78070 & 0.02663 & 0.1368 & 0.01872 & 2.479 & $0.0137^{*}$ \\
$\mathrm{mg}$ & 189.960 & 235.170 & 0.1499 & 0.02248 & 2.683 & $0.00768^{* *}$ \\
\hline
\end{tabular}

Table 9(c). Results of linear regression and t-test for mean monthly TCO and mean monthly SAI at Mombasa

\begin{tabular}{lllllll}
\hline TCO & intercept & slope & $\mathrm{r}$ & $\mathrm{r}^{2}$ & $\mathrm{t}$ value & $\operatorname{Pr}(>|\mathrm{t}|)$ \\
\hline ssn & 255.08534 & 0.03435 & 0.1974 & 0.03895 & 3.613 & $0.000351^{* * *}$ \\
$\mathrm{sf}$ & 252.73619 & 0.03678 & 0.2043 & 0.04175 & 3.737 & $0.000220^{* * *}$ \\
$\mathrm{mg}$ & 182.044 & 276.413 & 0.1894 & 0.03586 & 3.412 & $0.000729^{* * *}$ \\
\hline
\end{tabular}

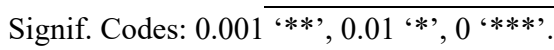


The straight line probabilistic model has slopes, intercepts and coefficient of determination values given in Table 9 (a) - (c) for the respective cities. The least square prediction equation is given as;

$$
\begin{array}{cc}
\text { Nairobi; } & \text { TCO }=252.81051+0.03362 \mathrm{ssn} \\
\mathrm{TCO}=250.64016+0.03490 \mathrm{sf} \\
\mathrm{TCO}=181.760+268.620 \mathrm{mg} \\
\text { Kisumu; } & \mathrm{TCO}=252.42988+0.02576 \mathrm{ssn} \\
& \mathrm{TCO}=250.78070+0.02663 \mathrm{sf} \\
\text { Mombasa; } & \mathrm{TCO}=189.960+235.170 \mathrm{mg} \\
\mathrm{TCO}=255.08534+0.03435 \mathrm{ssn} \\
\mathrm{TCO}=252.73619+0.03678 \mathrm{sf} \\
\mathrm{TCO}=182.040+276.413 \mathrm{mg}
\end{array}
$$

The significance of this linear dependence are justified by the $\mathrm{t}$ - test where the $\mathrm{t}$ - statistic value3.190, 3.195, 2.999 for ssn, sf and $\mathrm{mg}$ at Nairobi, 2.486, 2.479 and 2.683for ssn, sf and mg at Kisumu and 3.613, 3.737, 3.412 for ssn, sf and mg at Mombasa are greater than the t critical Table 9(a) - (c) at $\alpha=0.05$.

The linear dependence is further confirmed by the ANOVA results as the calculated F - values at the three cities and their corresponding SAI variables Table 10 (a) - (c) are greater than the critical F at $\alpha=0.05$.

Table 10(a). ANOVA table for Nairobi

\begin{tabular}{lll}
\hline TCO & F value & $\operatorname{Pr}(>F)$ \\
\hline ssn & 10.175 & $0.001564^{* *}$ \\
sf & 10.210 & $0.001535^{* *}$ \\
mg & 9.017 & $0.002926^{* *}$ \\
\hline
\end{tabular}

Table 10(b). ANOVA table for Kisumu

\begin{tabular}{lll}
\hline TCO & F value & $\operatorname{Pr}(>\mathrm{F})$ \\
\hline ssn & 6.1778 & $0.01344^{*}$ \\
sf & 6.1442 & $0.01370^{*}$ \\
mg & 3.861 & $0.007684^{* *}$
\end{tabular}

Table 10(c). ANOVA table for Mombasa

\begin{tabular}{lll}
\hline TCO & F value & $\operatorname{Pr}(>F)$ \\
\hline ssn & 13.051 & $0.0003515^{* * *}$ \\
sf & 13.966 & $0.0002201^{* * *}$ \\
$\mathrm{mg}$ & 10.050 & $0.0007293^{* * *}$ \\
\hline
\end{tabular}

Signif. Codes: $0.001^{* *}, 0.01^{*}, 0^{* * *}$.

Hence these results reveal that the mean monthly TCO at Nairobi, Kisumu and Mombasa have positive correlation with SAI variables. A linear relationship exists between the two variables in all the cities. The best fit equations 1(a) - (1c) for Nairobi, equations 2(a) - 2(c) for Kisumu and equations 3(a) - 3(c) for Mombasa suggest an increase in TCO of about $2-3 \%$ (Nairobi), $1-2 \%$ (Kisumu) and $3-4 \%$ (Mombasa) is attributed to solar activity indices; ssn, sf and mg. The contribution is not the same for all the SAI variables. The F10.7 cm solar radio flux seems to have the best input while the $\mathrm{Mg}$ II core to wing ratio has the least input in the variation of TCO at Nairobi, Kisumu and Mombasa. 


\subsection{Linear Multivariate Models}

A linear multivariate model of the form

$$
\mathrm{y}=b_{0}+b_{1} s s n+b_{2} s f+b_{3} m g
$$

Where y is the mean monthly TCO, $b_{0}, b_{1}, b_{2}$ and $b_{3}$ are the coefficients determined by the least square fittings. The coefficients are presented in the multivariate models 4 to 6 .

$$
\begin{aligned}
& T C O_{n r b}=382.58345+0.02036 s s n+0.07412 s f-508.24278 m g \\
& T C O_{k s m}=370.28900+0.01859 s s n+0.06200 s f-460.30874 m g \\
& T C O_{m s a}=356.70976+0.00279 s s n+0.08728 s f-405.00741 \mathrm{mg}
\end{aligned}
$$

Models in equations 4 to 6 shows solar forcing of TCO at the cities under study. Models in equations 4 and 5 show direct forcing of sf and ssn, but a negative forcing of $\mathrm{mg}$ at Nairobi and Kisumu. All the models indicate direct forcing of sf and inverse forcing of mg at Nairobi, Kisumu and Mombasa. A direct forcing of ssn is indicated only at Nairobi and Kisumu. The high coefficients of $\mathrm{mg}$ in the models indicate that forcing due to the chromosphere and hence the solar ultraviolet radiation is more prominent on TCO during this study period compared to the photosphere and corona (Ndeda, Rabiu, Ngoo \& Ouma, 2010).

Table 11. Multiple correction coefficients results

\begin{tabular}{cllll}
\hline Model equation & $\mathrm{R}$ & $\mathrm{R}^{2}$ & $\mathrm{p}$ \\
\hline 4 & 0.196825 & 0.03874 & $0.005425^{* * *}$ \\
5 & 0.160000 & 0.025600 & $0.03995^{*}$ \\
6 & 0.218128 & 0.04758 & $0.001354^{* *}$ \\
\hline
\end{tabular}

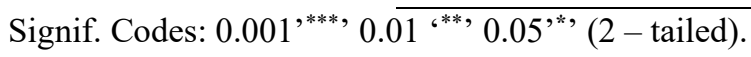

The multiple correlation coefficients, $\mathrm{R}$ and the significant levels for the three cities are shown in Table 11. At Nairobi $(\mathrm{R}=0.196825, \mathrm{p}=0.01)$, Kisumu $(\mathrm{R}=0.1600, \mathrm{p}<0.05)$ and Mombasa $(\mathrm{R}=0.218128, \mathrm{p}=0.001)$ are obtained. Hence $4 \%, 3 \%$ and $5 \%$ of the TCO at Nairobi, Kisumu and Mombasa can be predicted by the SAI variables.

\section{Conclusion}

A decreasing trend at significant levels of SAI variables is observed showing that, during the study period, SAI is decreasing with highest decrease in ssn $(94 \%$ of the mean), followed by sf $(34 \%)$ and least in $\mathrm{mg}(\approx 2 \%)$. Pearson correlation analysis demonstrated that both TCO and the SAI variables time series are highly correlated amongst each other. TCO are highly correlated with each other at $(0.936<\mathrm{r}<0.955, \mathrm{p}<0.001,2$ - tailed). SAI variables are also highly correlated with each other at $(0.941<\mathrm{r}<0.976, \mathrm{p}<0.001,2$ - tailed). Monthly mean SAI variables are significantly positively correlated with monthly mean TCO over the study period except for mean monthly TCO and averaged mg at Kisumu is not significant $(\mathrm{p}>0.05)$

All correlations are positive hence TCO and SAI are more or else in phase. The highest correlation is found to exist between SAI variables and mean monthly TCO at Mombasa with ssn (0.1974), sf (0.2039) and mg (0.1740). Among the SAI variables, sf and then ssn, seems to have more contribution (input) in ozone production in Nairobi and Mombasa while ssn and then sf, has more input in ozone production in Kisumu. Mg II core to wing ratio has the least contribution to ozone in Nairobi and Mombasa but insignificant in Kisumu.

The mean monthly TCO and mean monthly SAI variables have correlations at both long and short lags. Positive maximum correlation is again observed between $\mathrm{TCO}$ at all the cities and SAI variables. A local maximum cross correlation between mean monthly TCO and mean monthly SAI variables is observed when ssn and sf variables are at month zero lag with TCO at all the cities. Therefore in all the cities, sf has an immediate impact and $\mathrm{mg}$ has a delayed impact on TCO while ssn has immediate impact on TCO at Nairobi and Mombasa but a delayed impact at Kisumu.

A weak linear relationship exists between the two variables in all the cities. TCO and SAI bore a significant linear relationship at $5 \%$. An increase in TCO of about $2-3 \%$ (Nairobi), $1-2 \%$ (Kisumu) and $3-4 \%$ (Mombasa) is attributed to solar activity indices. The contribution is not the same for all the SAI variables. The F10.7 cm solar radio flux seems to have the best input while the Mg II core to wing ratio has the least input in the variation of TCO at Nairobi, Kisumu and Mombasa. 
All cities indicate positive forcing of the coronal index on TCO. The solar activity in the chromosphere through the $\mathrm{mg}$, has negative forcing of TCO in all the cities. The index of the photosphere, the ssn, has positive forcing of TCO at Nairobi and Kisumu and a negative forcing at Mombasa. The multiple correlation coefficients and significant levels of $(\mathrm{R}=0.196825, \mathrm{p}=0.01)$ at Nairobi, $(\mathrm{R}=0.1600, \mathrm{p}<0.05)$ at Kisumu and $(\mathrm{R}=0.218128, \mathrm{p}=$ 0.001 ) at Mombasa are obtained showing $4 \%, 3 \%$ and $5 \%$ of the TCO at Nairobi, Kisumu and Mombasa can be predicted by the SAI variables.

\section{Acknowledgements}

We would like to thank Greg Bodeker and Jan Markus Diezel for providing the combined total column ozone database for the three Kenyan cities. The authors are thankful to the NOAA and NASA team for the solar indices data (international sunspot number, the $\mathrm{F} 10.7 \mathrm{~cm}$ solar radio flux and the $\mathrm{Mg}$ II core to wing ratio. We are also grateful to the National Commission for Science, Technology and Innovation (NACOSTI) - the research authorizing body in Kenya, for provision of funds that enabled us to undertake this research. Finally, the authors wish to express their sincere thanks to the anonymous reviewers for providing constructive comments and suggestions to enhance the quality of the article.

\section{References}

Angell, J. K. (1989). On the relation between atmospheric ozone and sunspot number. J Clim (USA), 2, 1404-1416. http://dx.doi.org/10.1175/1520-0442(1989)002<1404:OTRBAO >2.0.CO;2

Bodeker, G. E., Hassler, B., Young, P. J., \& Portmann, R. W. (2013). A vertically resolved, global, gap-free ozone database for assessing or constraining global climate model simulations. Earth System Science Data, 5, 31$43 \mathrm{http}: / /$ dx.doi.org/10.5194/essd-5-31-2013

Bruevich, E. A., Bruevich, V. V., \& Yakunina, G. V. (2013). Correlational study of some solar activity indices in the cycles 21 - 23. Retrieved from arXiv preprint arXiv: 1304. 4545, 2013 - arxiv.org

Charbonneau, P. (2005). Dynamo models of the solar cycle. Living Reviews in Solar Physics, 7(2), 20. http://dx.doi.org/10.12942/lrsp-2005-2

Covington, A. E. (1948). Solar noise observations on 10.7 centimeters. Royal Astronomical Society of Canada Journal, 48(4), 136. http://dx.doi.org/10.1109/jrproc.1948.234598

de Toma, G., White, O. R., Knapp, B. G., Rottman, G. J., \& Woods, T. N. (1997). Mg II core-to-wing index: Comparison of SBUV2 and SOLSTICE time series. J. Geophysical. Res., 102(A2), 2597-2610. http://dx.doi.org/10.1029/96JA03342

Dudok de Wit, T., \& Watermann, J. (2009). Solar forcing of the terrestrial atmosphere; Space Physics (physics. pace- ph). Atmospheric and Oceanic Physics (physics.ao-ph).

Dudok de Wit, T., Kretzschmar, M., Lilensten, J., \& Woods T. N. (2009). Finding the best proxies for the solar UV irradiance. Geophysical Research Letters 36, L10107. http://dx.doi.org/10.1029/2009g1037825

Floyd, L., Tobiska, W. K., \& Cebula, R. P. (2002). Solar UV irradiance, its variation, and its relevance to the Earth. Advances in Space Research, 29, 1427-1440. http://dx.doi.org/10.1016/S0273-1177(02)00202-8

Geller, M. A. (1988). Solar cycles and the atmosphere. Nature, 332, 584-585.

Gray, L. J. (2010). Stratospheric equatorial dynamics. In L. Polvani, A. H. Sobel, \& D. W. Waugh (Eds.), The stratosphere: Dynamics, transport, and chemistry, volume 190 (pp. 93-108). Washington DC: American Geophysical Union. http://dx.doi.org/10.1029/2009GM000868

Gray, L. J., Rumbold, S. T., \& Shine, K. P. (2009). Stratospheric temperature and radiative forcing response to 11year solar cycle changes in irradiance and ozone. Journal of the Atmospheric Sciences, 66, 2402-2417. http://dx.doi.org/10.1175/2009JAS2866.1

Hathaway, H. D., Dibyendu, N., Wilson Robert, M., \& Reichmann, E. J. (2003). Evidence that a deep meridional flow sets the sunspot cycle period, The Astrophysical Journal, 589, 665- 670. http://dx.doi.org/10.1086/ 374393

Heath, D. F., \& Schlesinger, B. M. (1986). The $280 \mathrm{~nm}$ doublet as a monitor of changes in solar ultraviolet irradiance. J. Geophys. Res., 91, 8672-8682. http://dx.doi.org/10.1029/JD091iD08p08672

Jana, P. K.., Bhattacharyya, S., \& Midya S. K. (2013). Equatorial, tropical and Antarctic ozone depletion and heir correlation with relative sunspot numbers. Int. J. Res. Chem. Environ., 3, 48-61. 
Labitzke K., \& Van Loon, H. (1997). Total ozone and the 11-yr sunspot cycle. J. Atmos Sol-Terr Phys (UK), 59(1) 9-19. http://dx.doi.org/10.1016/S1364-6826(96)00005-3

Lean, J. (2000b). Short term, direct indices of solar variability. Space Science Reviews, 94, 39-51. http://dx.doi.org/10.1023/A:1026726029831

Lilensten, J., Dudok de Wit, T., Kretzschmar, M., Amblard, P.-O., Moussaoui, S., Aboudarham, J., \& Auch`ere, F. (2008). Review on the solar spectral variability in the EUV for space weather purposes. Annales Geophysicae, 26, 269-279. http://dx.doi.org/10.5194/angeo-26-269-2008

Lockwood, M. (2004). Solar outputs, their variations and their effects of Earth. In I. Redi, M. G"udel, \& W. Schmutz (Eds.), The sun, solar analogs and the climate (pp. 107-304). Berlin, Heidelberg, New York: Springer Proceedings of Saas-Fee Advanced Course, 34.

Marsh, D. R., Garcia R., Kinnison, D., Boville, B., Sassi, F., Solomon, S. C., \& Matthes, K. (2007). Modeling the whole atmosphere response to solar cycle changes in radiative and geomagnetic forcing. Journal of Geophysical Research, 112, D23306. http://dx.doi.org/10.1029/2006JD008306

Ndeda, J. H. O., Rabiu, A. B., Ngoo, L. H. M., \& Ouma, G. O. (2010). Estimation of climatic parameters from solar indices using ground based data from Kenya, East Africa. Journal of Science and Technology, 31(1), 131.

Newman, P. A. (2004). Stratospheric ozone. Retrieved fom: http://www. ccpo.odu.edu/SEES/ozone/class/ hapter_1/index.htm

Obiekezie, T. N. (2009). Sunshine activity and total column ozone variation in Lagos, Nigeria. Moldavian Journal of the Physical Sciences, 8(2), 169-172.

Rabiu, B., \& Omotosho, T. V. (2003). Solar activity and TCO variation in Lagos, Nigeria. Nigeria Journal of Pure and Appl. Physics, 2(1), 17-20. http://dx.doi.org/10.4314/njpap.v2i1.21433

Selvaraj, R. S., Gopinath, T., \& Jayalakshmi, K. (2010b). Statistical relationship between surface ozone and solar activity in a tropical rural coastal site, India. Indian Journal of Science and Technology, 3(7), 792- 794.

Songa, C. M. M., Ndeda, J., \& Ouma, G. (2015). Total column ozone variability and trends over Kenya using combined multiple satellite - based instruments, Applied Physics Research, 7(5), 87-101. http://dx.doi.org/10.5539/apr.v7n5p87

Spruit, H. C. (2000). Theory of solar irradiance variations. Space Sci. Rev., 94, 113-126. http://dx.doi.org/10. 1023/A:1026742519353

Suess, K., Snow, M., Viereck, R., \& Machol, J. (2016). Solar spectral proxy irradiance from GOES (SSPRING): a model for solar EUV irradiance. J. Space weather climate, 6, A10. http://dx.doi.org/10.1051/swsc/2016003

Tapping, K. F. (2013). The $10.7 \mathrm{~cm}$ solar radio flux (F10.7). Space weather, 11, 394 - 406. http://dx.doi.org/10. 1002/swe.20064

Tobiska W., Bouwer S., \& Bowman B. (2008). The development of new solar indices for use in thermospheric density modeling. Journal of Atmospheric and Solar-Terrestrial Physics, 70, 803-819. http://dx.doi.org/10. 1016/j.jastp.2007.11.001

Tsiropouli, G. (2003). Signatures of solar activity variability in meteorological parameters. Journal of Atmospheric and Solar-Terrestrial Physics, 65, 469-482. http://dx.doi.org/10.1016/S1364-6826(02)00295-X

Viereck, R. A., Puga, L., MacMullin, D., Judge D., Weber, M., \& Tobiska W. K. (2001). The Mg II Index: A proxy for Solar EUV. Letters, 28(7), 1343-1346. http://dx.doi.org/10.1029/2000g1012551

\section{Copyrights}

Copyright for this article is retained by the author(s), with first publication rights granted to the journal.

This is an open-access article distributed under the terms and conditions of the Creative Commons Attribution license (http://creativecommons.org/licenses/by/3.0/). 